\title{
Random Telegraph Signal phenomena in avalanche mode diodes: Application to SPADs
}

\author{
V. Agarwal, A.J. Annema, S. Dutta, R.J.E. Hueting, L.K. Nanver, B. Nauta \\ University of Twente, Enschede, The Netherlands \\ Email: v.agarwal@utwente.nl
}

\begin{abstract}
The current-voltage ( $I V)$ dependency of diodes close to the breakdown voltage is shown to be governed by Random Telegraph Signal (RTS) phenomena. We present a technology independent approach to accurately characterize the bias dependent statistical RTS properties and show that these can fully describe the steep $I V$-dependency in avalanche. The statistical properties also allow to more accurately describe e.g. the value of the self sustaining avalanche current that is crucial in designing optical detection systems using avalanche photo diodes or single photon avalanche diodes (SPADs). More accurate modelling is shown to allow improving on e.g. count rates, dead time and afterpulsing in quenching and recharge circuits for SPADs. Measurements are performed on diodes in a $140 \mathrm{~nm}$ SOI CMOS technology.
\end{abstract}

Keywords - Self-sustaining avalanche current, Latching current, RTS, Random Telegraph Noise, SPAD, Avalanche breakdown, Breakdown voltage

\section{INTRODUCTION}

The triggering phenomenon of avalanche in diodes has been described in [1]-[3]. Although most applications treat avalanche as the limiting region for using diodes, some applications explicitly make use of the avalanche region as the operating region. Major applications include optical detectors using avalanche photo diodes (APDs) or single photon avalanche diodes (SPADs), see e.g. [4].

APDs and SPADs are $\mathrm{p}-\mathrm{n}$ junction diodes that are reverse biased at a voltage $V_{\mathrm{R}}$ near and above their breakdown voltage $V_{\mathrm{BR}}$ respectively. In that region, the electric field is so high that a single free carrier, e.g. generated by a photon, can trigger avalanche by impact ionization and where the avalanche current increases swiftly to macroscopic values. In APDs finite avalanche multiplication factors are used, while SPADs are operated in Geiger mode and require a quenchand-recharge circuit to externally quench avalanching once triggered and to reset the diodes for subsequent photon detection [4]. This results in highly sensitive optical sensors with excellent time resolution and produces an output signal that can easily be detected. SPADs have found many applications in areas such as positron emission tomography, single photon emission computed tomography [4]. In this paper we focus on the avalanche process and applications to SPADs.

Quenching of diodes in avalanche is not well described and as a rule-of-thumb, it is reported that the avalanche is self sustaining for diode currents $I_{\mathrm{D}}$ higher than $100 \mu \mathrm{A}$ (denoted as latching current) [5],[6]. Below this latching current, there is a high probability that avalanche is quenched and then all free

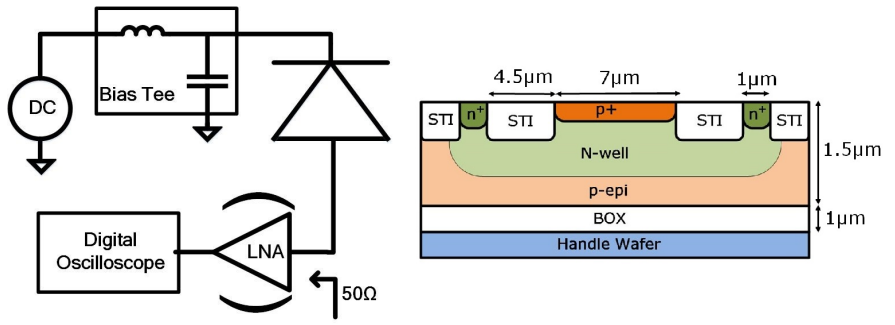

Fig. 1: Experimental Setup for wafer probing measurements (left), and a cross-section of the diode (right). The low-noise amplifier (LNA) is used only at low current levels.

carriers recombine. In passive quenching circuits, as another rule-of-thumb, typically a $50 \mathrm{k} \Omega$ per extra volt of excess bias $V_{\mathrm{EX}}=V_{\mathrm{R}}-V_{\mathrm{BR}}$ is used as a quenching resistance $\left(R_{\mathrm{Q}}\right)$ [5]. However, experimentally $V_{\mathrm{BR}}$ is ill defined in literature which also renders $V_{\mathrm{EX}}$ ill defined; we therefore introduce a unique definition of voltages that limit the steep $I V$ part in avalanche. We show that these generally accepted rulesof-thumb to estimate e.g. $R_{\mathrm{Q}}$ can yield an overestimation for high counting rate applications such as optical links and optical interconnects [7].

This paper is outlined as follows. An experimental setup is described in section II that enables us to achieve very low external quenching, limited by the $50 \Omega$ input resistance of the measurement setup. This setup enables measuring currents with $160 \mathrm{nA}$ current resolution and $100 \mathrm{ps}$ time resolution which is sufficient to accurately measure and model avalanche Random Telegraph Signal (RTS) processes. Analyses show that $I_{\mathrm{D}}$ near breakdown can be characterized as RTS. We then describe a time domain method to analyze the RTS signals in section III. In section IV, we present the experimental results, analyze the RTS and discuss the underlying statistics. These analyses allow to extract bias dependent statistical RTS properties such as expected values for the RTS magnitude and RTS duty cycle as a function of $V_{\mathrm{R}}$. Combined, these are shown to fully describe the steep $I V$ dependency in avalanche. Using the results, a parametric self sustaining avalanche current level can be defined which enables accurate design of e.g. active or passive quench-and-recharge circuits. In section $\mathrm{V}$, as an application example we use these results to more precisely estimate the $R_{\mathrm{Q}}$ in a passive quench circuit. 


\section{EXPERIMENTAL SETUP}

Fig. 1 shows the experimental setup we used to characterize diodes in a $140 \mathrm{~nm}$ CMOS SOI technology [8]; the schematic cross section of the diode is also shown in the figure. The multiplication (or depletion) region of the diode is beneath the $p^{+}$region [9]. In our setup, there are only non optical sources of free carriers to trigger avalanche in the diode: either from thermal generation, diffusion or defects in the multiplication region [10]. However, optical sources (photons) can also trigger avalanche. Once triggered, the avalanche contribution $I_{\mathrm{A}}$ in the total diode current $I_{\mathrm{D}}$ flows until (actively, passively or self) quenched and only after that the diode reverts to its original non-avalanching state.

In this paper, the main focus is on the characterization and modeling of self-sustaining properties of $I_{\mathrm{A}}$. For that reason, the total $R_{\mathrm{Q}}$ was minimized, here to only $50 \Omega$ of the measurement setup. This was accomplished by low-ohmically biasing the cathode using a bias tee and by shunting the anode by the $50 \Omega$ input resistance of a high performance oscilloscope (Agilent DSO54854A). At low RTS current magnitude levels an additional high-bandwidth low-noise amplifier (LNA) was used in front of the oscilloscope input; also this amplifier has $50 \Omega$ input resistance. A high data acquisition rate of $5 \mathrm{GS} / \mathrm{s}$ ensures that very narrow pulses could also be detected. This setup allows measuring currents with $160 \mathrm{nA}$ resolution with a noise floor of $0.4 \mathrm{nA} / \sqrt{\mathrm{Hz}}$. Measurements were done in a Faraday's cage in complete dark conditions at a temperature of $298 \mathrm{~K}$ using wafer probing methods. The data was acquired for a total duration of $1 \mathrm{~ms}$ at each bias condition.

Fig. 2 shows the DC current-voltage (IV) characteristics as measured by a sense-and-measurement unit (SMU) of a Keithley B2901A, using $1 \mathrm{~s}$ integration time; a micrograph of the diode is shown as inset. In section IV it is shown that the steep part of the DC IV curve is fully described by bias dependent statistical properties of the RTS underlying the avalanche process; also an exact determination of $V_{\mathrm{BR}}$ is given in section IV. The measurements indicate that the avalanche process starts around $14.7 \mathrm{~V}$ with $I_{\mathrm{A}}$ rising sharply between $14.8 \mathrm{~V}$ and $14.9 \mathrm{~V}$; a major part of this paper focusses on that voltage range.

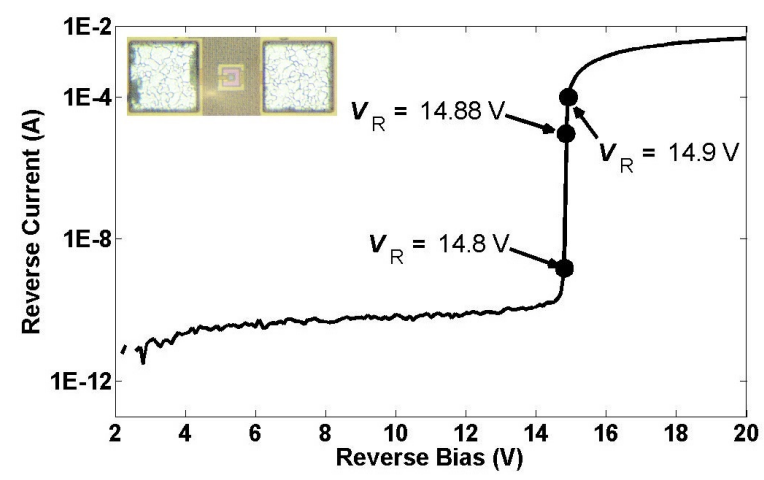

Fig. 2: The DC characterized $I V$ characteristics and the micrograph of the diode (inset).

\section{RTS ANALYSIS PROCEDURE}

An excerpt of a measured waveform at $V_{\mathrm{R}}=14.88 \mathrm{~V}$ is shown in Fig. 3. It can be seen that the $I_{\mathrm{A}}$ flows in the form of current pulses having more or less two discrete levels having random pulse duration. These current pulses were observed consistently among the several samples of this diode structure. This type of behavior was reported as unstable microplasma behavior of diodes near $V_{\mathrm{BR}}$ [1],[11],[12]. However, a complete time domain analysis to extract statistical properties and linking those to DC-observed behavior and quenching was not presented earlier.

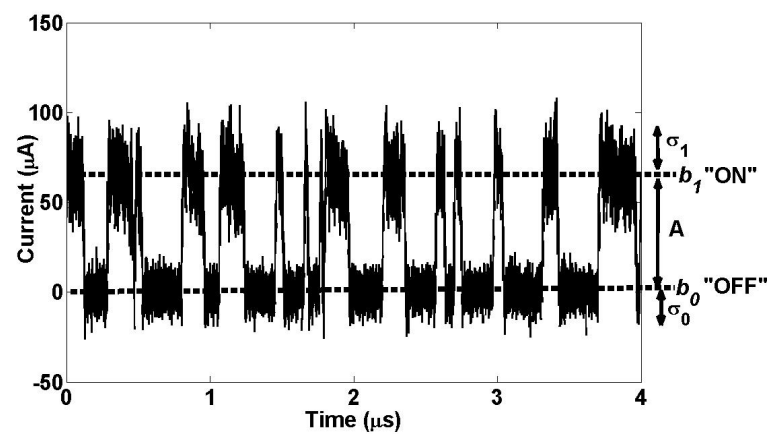

Fig. 3: An excerpt of the avalanche current at $V_{\mathrm{R}}=14.88 \mathrm{~V}$, showing avalanche current in the form of pulses of fixed amplitude and random duration.

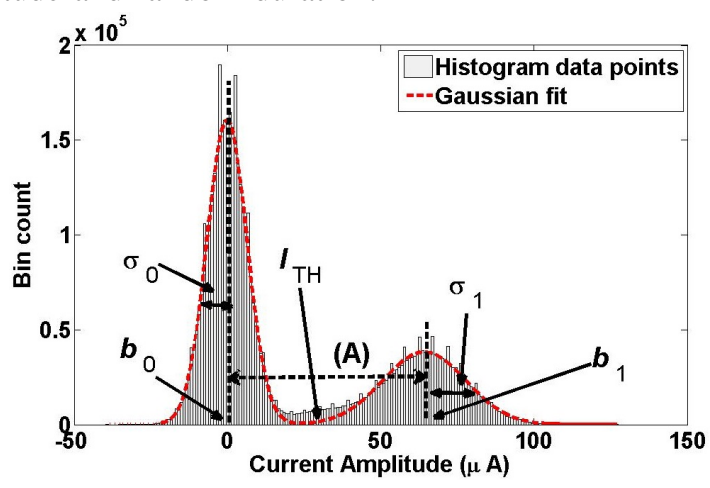

Fig. 4: Histogram of $I_{\mathrm{D}}$ showing OFF-level average $b_{0}$ and standard deviation $\sigma_{0}$, and showing ON-level average $b_{1}$ and variance $\sigma_{1}$. For time domain analyses a threshold level $I_{\mathrm{TH}}$ is used to optimally separate $\mathrm{ON}$ and OFF pulses.

It can be observed from switching between an $\mathrm{ON}$ and $\mathrm{OFF}$ level of $I_{\mathrm{A}}$ at fixed bias condition, that the avalanche process is not self sustaining and quenches itself with a specific turnoff probability [1],[2]. This ON-OFF behavior can be described by a RTS switching between the on-state and off-state current level [13]. The on-state of the avalanche is labeled as "ON" and the off-state of the avalanche is labeled as "OFF" in this work. RTS processes have a Poisson-distribution like nature and hence can be characterized by a few parameters:

- the expected ON-state lifetime $E\left(T_{\mathrm{ON}}\right)$

- the expected OFF-state lifetime $E\left(T_{\mathrm{OFF}}\right)$

- the amplitude difference between the states $A$ 
In the frequency domain, this type of Poisson distribution has a Lorentzian power spectral density [13],[14]; we focus only on time domain analyses as it allows to extract properties more easily. Part of the analysis procedure is illustrated in Fig. 4 [15], [16 $]^{1}$.

To obtain the RTS amplitude $A$, the time domain current $I_{\mathrm{D}}$ is displayed as a histogram. Using Gaussian fits, the mean values of the OFF-level $\left(b_{0}\right)$ and of the ON-level $\left(b_{1}\right)$ of the avalanche pulse can be determined, yielding $A=b_{1}-b_{0}$. In our measurement setup, the magnitude variance $\sigma_{0}$ is mainly due to the measurement setup, while $\sigma_{1}$ is mainly due to the RTS behavior of $I_{\mathrm{D}}$ in the steep part of the $I V$-curve.

To estimate purely the statistical properties in time domain, $I_{\mathrm{D}}$ is quantized into a pure two-level RTS using a simple level-crossing algorithm, using a threshold level $I_{\mathrm{TH}}$ that allows for optimum discrimination between the $\mathrm{ON}$ and $\mathrm{OFF}$ states, similar to common practice in e.g. data recovery in digital communication channels. With this, the measured $I_{\mathrm{D}}$ is quantized into $I_{\mathrm{Q}, \mathrm{RTS}}$ as:

$$
I_{\mathrm{Q}, \mathrm{RTS}}= \begin{cases}\mathrm{A}, & \text { if } I_{\mathrm{D}} \geq I_{\mathrm{TH}} \\ 0, & \text { otherwise }\end{cases}
$$

\section{EXTRACTING BIAS DEPENDENT RTS PROPERTIES}

Using the procedure described in section III, statistical properties of both the RTS magnitude and of the RTS switching can be obtained for various values of $V_{\mathrm{R}}$. In the context of avalanche processes and SPADs, the pulse width of RTS pulses, the inter-arrival time between RTS pulses and the RTS magnitude are the most relevant. Also the standard deviation of these give information, but is left out for paper-length reasons.

From $I_{\mathrm{Q}, \mathrm{RTS}}$, inter-arrival times for the ON and OFF state pulses are calculated. An example of a measured probability density function (PDF) of the inter-arrival time for $V_{\mathrm{R}}=14.88$ $\mathrm{V}$ is shown in Fig. 5; similar PDFs were obtained for other bias conditions in avalanche. These PDFs show that the inter-arrival times for the ON-state and OFF-state states are exponentially distributed which confirms that the observed RTS process (number of RTS pulses per unit time) is similar to a Poisson distribution [17]. The peak in the PDF at the far left hand side is because of the afterpulsing [9]. The conventionally used inter-arrival time for a certain state equals the lifetime for the other state.

Using PDFs as shown in Fig. 5, the expected lifetime in each state and the inter-arrival time at several values of $V_{\mathrm{R}}$ can now easily be calculated. Fig. 6 shows these obtained expected ON pulse width $E\left(T_{\mathrm{ON}}\right)$, the expected $\mathrm{OFF}$ width $E\left(T_{\mathrm{OFF}}\right)$. Note: $E\left(T_{\mathrm{ON}}\right)+E\left(T_{\mathrm{OFF}}\right)$ equals the expected pulse interarrival time which is the reciprocal of the expected RTS pulse repetition rate.

Combining the $E\left(T_{\mathrm{ON}}\right)$ and $E\left(T_{\mathrm{OFF}}\right)$ allows to derive an expected duty cycle $E(d)$ of the RTS current pulses in avalanche as $E(d)=E\left(T_{\mathrm{ON}}\right) /\left(E\left(T_{\mathrm{ON}}\right)+E\left(T_{\mathrm{OFF}}\right)\right)$. From

\footnotetext{
${ }^{1}$ In [15] and [16] a similar approach was used to characterize RTS phenomena in MOS transitors and in thin films.
}
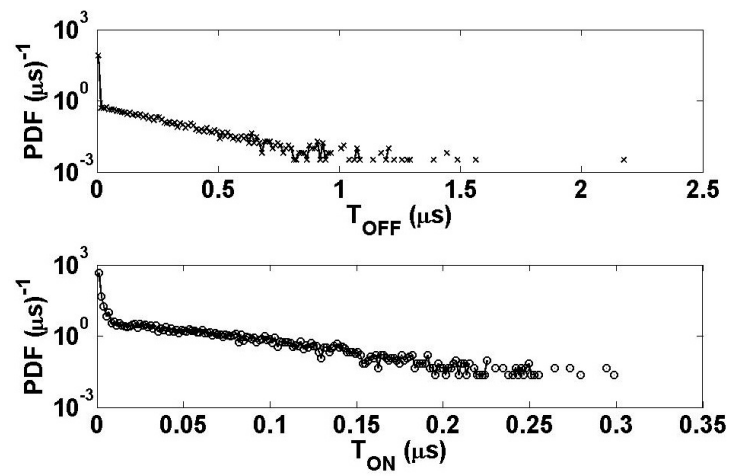

Fig. 5: The PDF of the inter-arrival times for the ON and OFF states at $14.88 \mathrm{~V}$; the PDFs have different $\mathrm{X}$-axis scales.

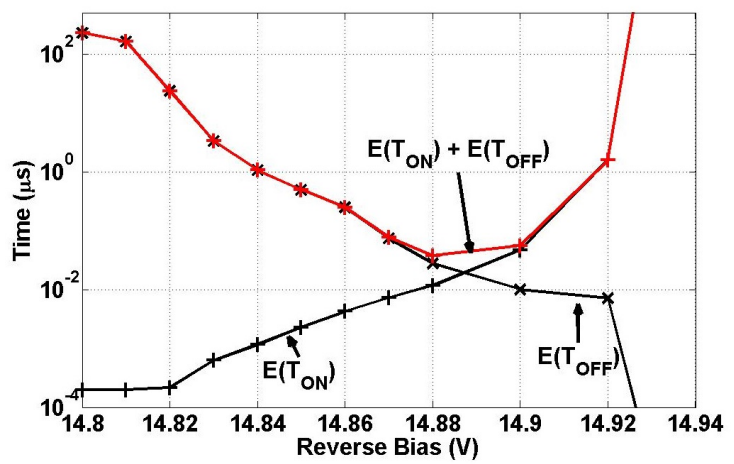

Fig. 6: Expected lifetimes (in $\mathrm{ON}$ and $\mathrm{OFF}$ states) and their sum as a function of $V_{\mathrm{R}}$. At $V_{\mathrm{R}}>14.92 \mathrm{~V}$, avalanche never quenches.

the statistics on the RTS magnitude we can readily derive $E(A)$. Fig. 7 shows both $E(d)$ and $E(A)$ as a function of $V_{\mathrm{R}}$, where $E(d)$ is shown logarithmically because of its large dynamic range.

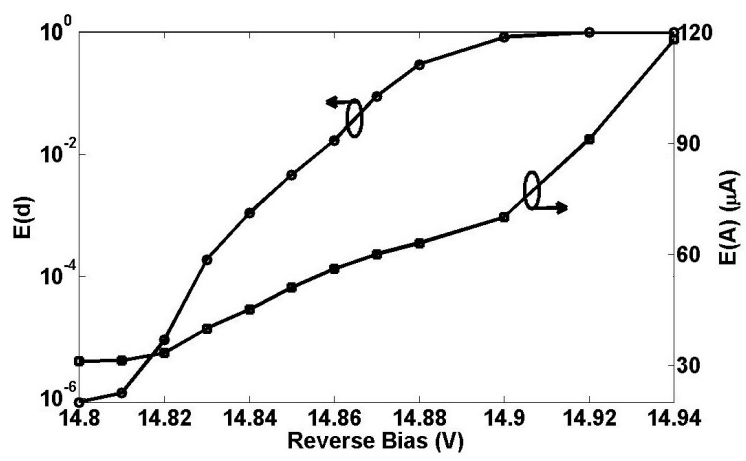

Fig. 7: RTS duty cycle $E(d)$ and magnitude $A$ as a function of reverse bias; $E(d)$ is on a semi-log scale.

Fig. 2 shows the DC-measured $I V$ curve of the diode below and in avalanche. Fig. 3 shows that in the steep part of the IVcurve the diode current $I_{\mathrm{D}}$ exhibits RTS behavior, confirmed by analyses on statistical properties in this section derived from measurements. Now, combining the bias dependent statistical 
properties of the RTS we can derive a $E(d)$-weighted RTSmagnitude $E(A)$ current $I_{\mathrm{RTS}}\left(V_{\mathrm{R}}\right)=E(d) \cdot E(A)$ that models the mere average effect of RTS avalanche pulses in $I_{\mathrm{D}}$. Fig. 8 shows this duty-cycle-weights RTS magnitude current as a function of $V_{\mathrm{R}}$ with the DC-measured diode current, showing very good correspondence. Note that in Fig. 8 the $\mathrm{x}$-axis is stretched around the avalanche region.

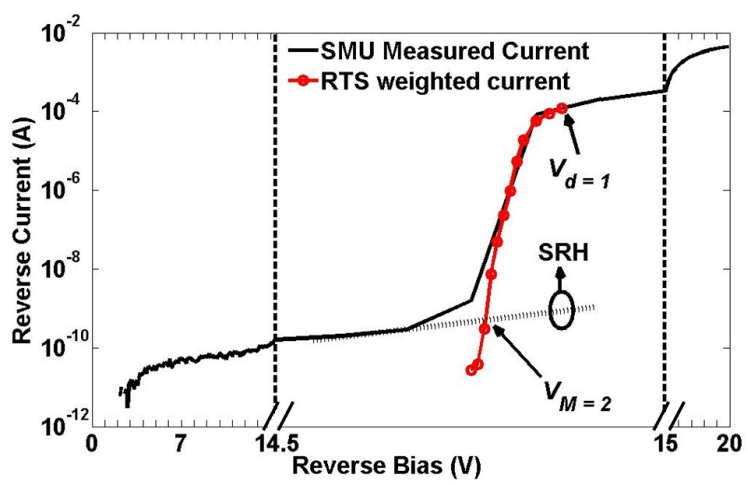

Fig. 8: DC-measured $I V$-curve and RTS weighted $E(d)$. $E\left(I_{\mathrm{ON}}\right)-V$-curve (for $V_{\mathrm{M}=2}<V_{\mathrm{R}}<V_{\mathrm{d}=1}$ ). For visibility reasons the $\mathrm{x}$-axis is stretched around the avalanche region.

As mentioned in section $\mathrm{I}$, the breakdown voltage $V_{\mathrm{BR}}$ is ill-defined in literature. However, using avalanche RTS current contribution statistics, we can uniquely define two voltages that delimit the steep part in the diode $I V$-curve due to avalanche. At the onset of avalanche, the voltage at which the contribution due to impact ionization $I_{\mathrm{A}}$ equals the SRHleakage component will be denoted $V_{M=2}$; at this voltage the multiplication factor $M=2$. The steep part of the $I V$ curve is mainly due to the strong bias dependency of $E(d)$; this is upper limited at $E(d)=1$ at voltage $V_{\mathrm{R}}=V_{\mathrm{d}=1}$; both are included in Fig. 8.

\section{APPLICATION IN SPADS}

Bias dependent statistical properties of the RTS can be used to accurately calculate the required value of a quenching resistance $R_{\mathrm{Q}}$ in passive quench circuits. For example, for an expected $5 \%$ duty cycle (or $95 \%$ probability of self quenching), for our $140 \mathrm{~nm}$ SOI CMOS technology the $V_{\mathrm{R}}$ at these quench conditions is $V_{\mathrm{Q}}=14.87 \mathrm{~V}$ and $E\left(I_{\mathrm{ON}}\right) \approx 60 \mu \mathrm{A}$. Hence, then $R_{\mathrm{Q}}$ should be $16 \mathrm{k} \Omega$ per extra voltage of $V_{\mathrm{EX}}=V_{\mathrm{R}}-V_{\mathrm{Q}}$. The traditional approach would suggest us to use a $R_{\mathrm{Q}}=50 \mathrm{k} \Omega \cdot\left(V_{\mathrm{R}}-V_{\mathrm{BR}}\right)$ [5] while using the conventional SMU measured $I V$ characteristics, $R_{\mathrm{Q}}>100 \mathrm{k} \Omega \cdot\left(V_{\mathrm{R}}-V_{\mathrm{BR}}\right)$ using an ill-defined $V_{\mathrm{BR}}$.

TABLE I: Quenching Resistance $\propto 1 /$ count rate

\begin{tabular}{|l|l|l|l|}
\hline & $\begin{array}{l}\text { RTS Approach } \\
(5 \% E(d))\end{array}$ & SMU-IV Âpproach & $\begin{array}{l}\text { Traditional } \\
\text { Approach }\end{array}$ \\
\hline$R_{\mathrm{Q}}(k \Omega)$ & $16\left(V_{\mathrm{R}}-V_{\mathrm{Q}}\right)$ & $>100\left(V_{\mathrm{R}}-V_{\mathrm{BR}}\right)$ & $50\left(V_{\mathrm{R}}-V_{\mathrm{BR}}\right)$ \\
\hline
\end{tabular}

In passive quench circuits, the quenching time $\left(\tau_{\mathrm{Q}}\right)$ is usually significantly lower than the recharge time $\tau_{\mathrm{R}}$ [6].
Then the $\tau_{\mathrm{R}}=C_{\mathrm{P}} R_{\mathrm{Q}}$ is the dominant factor limiting e.g. the count rate in SPADs. From table I, it can be seen that the count rate can be increased by a roughly a factor 3 to 6 compared to the $R_{\mathrm{Q}}$ calculated using traditional rule-of-thumbs approaches. Smaller $R_{\mathrm{Q}}$ also results in lower $C_{\mathrm{P}}$ and hence lower afterpulsing and dead time [6].

\section{CONCLUSION}

RTS phenomena in diode currents were shown to fully determine the steep $I V$-dependency in avalanche. Based on the time domain analysis of RTS phenomena in avalanche, we have presented a relatively simple and technology independent approach to determine many bias dependent RTS properties of which RTS duty cycle and magnitude are the more important. Using this, the value of self sustaining avalanche current in diodes can parametrically be determined, which can be used to e.g. calculate the quenching resistance in a passive quench circuit SPADs. It is shown that at least in our technology this can significantly increase count rates.

\section{ACKNOWLEDGMENT}

This work is funded by Dutch Technology Foundation (STW), an Applied Science division of NWO. The authors thank NXP Semiconductors for silicon donation. We also thank Henk de Vries and Gerard Wienk for technical support.

\section{REFERENCES}

[1] R. J. McIntyre, Theory of Microplasma Instability in Silicon, J. of Applied Physics 32, 983, 1961.

[2] R. H. Haitz, Model for the Electrical Behavior of a Microplasma, J. of Applied Physics 35, 13701964.

[3] W. G. Oldham et al, Triggering Phenomena in Avalanche Diodes, IEEE Transactions on Electron Devices, vol. ED-19, No. 9, 1972.

[4] Charbon E., Single-photon imaging in complementary metal oxide semiconductor processes, Phil. Trans. R. Soc. A 372: 20130100, 2014.

[5] S. Cova et al, Avalanche photodiodes and quenching circuits for singlephoton detection, Proc. IEEE 97, 1166-1185 2009.

[6] A. Gallivanoni et al., Progress in Quenching Circuits for Single Photon Avalanche Diodes, IEEE Transactions on nuclear science, vol. 57, no. 6, pp 3815-3826, 2010.

[7] D. A. B. Miller, Device requirements for optical interconnects to silicon chips, Proc. IEEE, vol. 97, no. 7, pp. 1166-1185, 2009.

[8] Myung-Jae Lee et al., A first single-photon avalanche diode fabricated in standard SOI CMOS technology with a full characterization of the device, Optics Express 13200, 2015.

[9] M. W. Fishburn, Fundamentals of CMOS single photon avalanche diodes, Ph.D. dissertation (Delft Univ. of Technology, The Netherlands, 2012).

[10] S. M. Sze and K. K. Ng, Physics of Semiconductor Devices, Third Ed. (Wiley, 2007).

[11] K. G. McKay, Avalanche Breadkdown in Silicon, Physical Review, pp. 877 - 884, vol. 94, no 4, 1954.

[12] K. S. Champlin, Microplasma Fluctuations in Silicon, J. of Applied Physics 30, 1039, 1959.

[13] S. Machlup, Noise in Semiconductors: Spectrum of a Two-Parameter Random Signal, J. Appl. Phys. 25, 341, 1954.

[14] M. A. Karami et al., RTS noise characterization in Single Photon Avalanche Diodes, IEEE Electron Device Letters,vol. 31, no 7, 2010.

[15] J.S. Kolhatkar Steady-state and cyclo-stationary RTS noise in MOSFETS, $\mathrm{PhD}$ thesis, University of Twente, The Netherlands, 2005.

[16] Y. Yuzhelevski et al. Random telegraph noise analysis in time domain, Review of Scientific Instruments 71, 16812000.

[17] Y. Kang et al. Dark count probability and quantum efficiency of avalanche photodiodes for single-photon detection, Appl. Phys. Lett. 83, 29552003. 